\title{
Overexpression of Rictor protein in colorectal cancer is correlated with tumor progression and prognosis
}

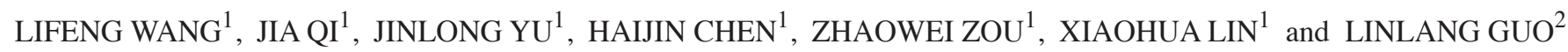 \\ Departments of ${ }^{1}$ General Surgery and ${ }^{2}$ Pathology, Zhujiang Hospital of \\ Southern Medical University, Guangzhou, Guangdong 510280, P.R. China
}

Received January 24, 2016; Accepted July 3, 2017

DOI: $10.3892 / \mathrm{ol} .2017 .6936$

\begin{abstract}
In order to understand the clinical significance of rapamycin-insensitive companion of mammalian target of rapamycin (Rictor) in colorectal cancer (CRC), 62 CRC tissue samples excised during operations were evaluated by immunohistochemistry. Analysis of the association between the expression level of Rictor protein and clinicopathological parameters demonstrated that the expression level of Rictor in CRC tissues was significantly higher than that in paracarcinoma tissues $(\mathrm{P}<0.0001)$. In cellular experiments, this result was further confirmed by comparing differences in Rictor expression between the CRC cell lines HCT116, SW480 and LoVo, and the human normal liver cell line HL-7702. It was also noticed that the expression of Rictor was associated with Dukes stage, lymphatic metastasis and prognosis, as determined by $\chi^{2}$ test, Kaplan-Meier analysis and log-rank test. These results suggest that Rictor may be a novel target for the treatment and prognostic assessment of CRC patients in the future.
\end{abstract}

\section{Introduction}

Colorectal cancer (CRC) is the third most commonly diagnosed cancer in the world and its morbidity has increased in recent years (1). Dysregulation of mammalian target of rapamycin (mTOR) and mTOR signaling is frequently observed in a variety of human cancers (2). mTOR exists in two functionally distinct complexes: mTOR complex 1 (mTORC1), containing mTOR and regulatory-associated protein of $\mathrm{MTOR}$, and $\mathrm{mTORC} 2$, containing mTOR and rapamycin-insensitive companion of mTOR (Rictor) (3). mTORC1 is sensitive to rapamycin and responds to multiple stimuli, including energy status, growth factors, amino acids and inflammation (4). mTORC2 affects

Correspondence to: Dr Jinlong Yu, Department of General Surgery, Zhujiang Hospital of Southern Medical University, 253 Industrial Avenue, Guangzhou, Guangdong 510280, P.R. China E-mail: 374320667@qq.com

Key words: colorectal cancer, rapamycin-insensitive companion of mammalian target of rapamycin, immunohistochemistry, western blotting cell morphology and actin polymerization $(5,6)$, and mainly promotes cell proliferation and survival through phosphorylation of Akt and serine/threonine-protein kinases (STK) $(7,8)$. Rictor, which is insensitive to rapamycin, forms mTORC2 by binding to mammalian lethal with SEC13 protein 8, mammalian stress-activated protein kinase interacting protein 1 and protein observed with Rictor (9). Currently, only a limited number of reports indicate that Rictor has certain biological functions in malignant tumors. For example, it has been reported that microRNA (miR)-152 acts as a tumor suppressor by targeting Rictor in gynecological cancers (10). Although Rictor may be involved in cancer progression, its expression in $\mathrm{CRC}$ remains unclear.

Thus, the present study evaluated the expression levels of Rictor in CRC tissue (experimental group) vs. paracarcinoma tissue (control group) using immunohistochemistry in $62 \mathrm{CRC}$ paraffin-embedded tissue samples excised during operations. The difference in expression was further verified at the cellular level by comparing the differences in the expression level of Rictor between CRC cells and human normal liver cells. The association between the expression levels of Rictor protein and the clinicopathological features of the two groups of patients was compared using the $\chi^{2}$ test, while the association between the expression of Rictor and the overall survival rate of CRC patients was analyzed using the Kaplan-Meier survival analysis method.

\section{Materials and methods}

Materials and chemicals. HCT116, SW480 and LoVo cells were obtained from the American Type Culture Collection (Manassas, VA, USA), which performed cell line authentication using DNA fingerprinting by short tandem repeat analysis. The normal human hepatocyte cell line HL-7702 was purchased from the Cell Bank of the Chinese Academy of Sciences (Shanghai, China).

RPMI-1640 medium, trypsin, EDTA, fetal bovine serum (FBS), penicillin and streptomycin were purchased from Gibco (Thermo Fisher Scientific, Inc., Waltham, MA, USA). Monoclonal anti-Rictor antibody (used for IHC and western blot analysis) produced in mouse (SAB5300210) was purchased from Sigma-Aldrich; Merck KGaA (Darmstadt, Germany). Alexa Fluor ${ }^{\circledR}$ 594-conjugated goat anti-mouse IgG secondary antibody (ZF-0513) was purchased from ZSGB-BIO Technology Co., Ltd. (Beijing, China), and used 

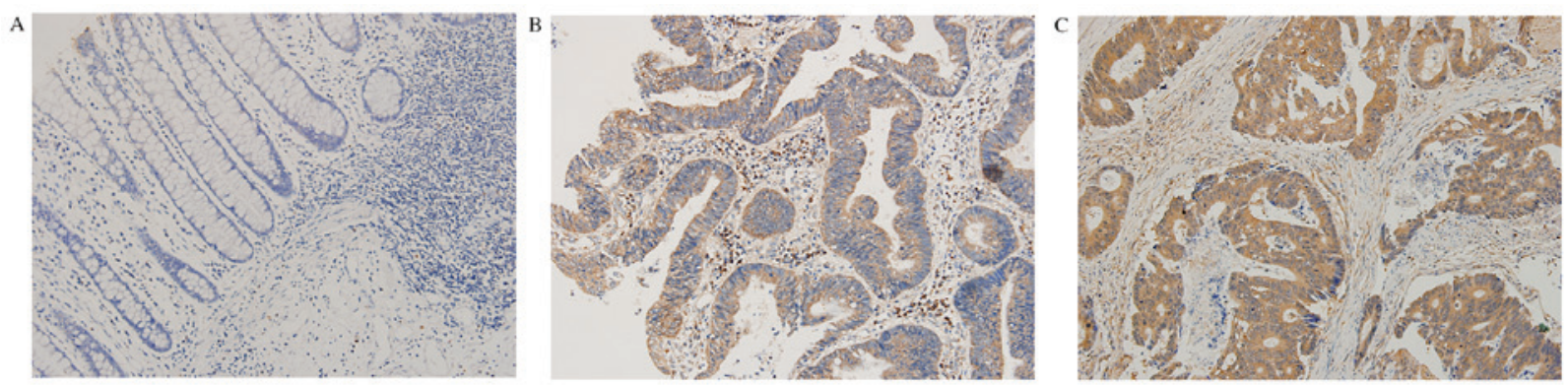

Figure 1. Rapamycin-insensitive companion of mammalian target of rapamycin expression in colorectal cancer and adjacent tissues. Representative images of (A) negative, (B) moderate and (C) strong staining. Original magnification, $\mathrm{x} 400$.

for IHC and western blot analysis. Anti- $\beta$-tubulin (MG7) mouse monoclonal antibody was acquired from Beijing Ray Antibody Biotech (RM2003; Beijing, China). Goat anti-mouse IgG was obtained from Beijing Biosynthesis Biotechnology Co., Ltd. (cat. no. bs-0296G; Beijing, China) and used for western blot analysis. ECL Western Blotting kit was acquired from Biyuntian Biotech Co., Ltd. (Shanghai, China).

Sample collection. A total of $62 \mathrm{CRC}$ paraffin-embedded tissue samples excised during operations were collected from Zhujiang Hospital Affiliated to Nanfang Medical University (Guangzhou, China) during June 2008 and August 2010. Patients included in the study did not receive any chemical treatment or other anticancer therapies prior to surgery. Patients excluded from the study were those who did not have complete follow-up or clinicopathological data, and those who did not provide informed consent for participation in the study, as required by the Medical Ethics Committee Affiliated to Zhujiang Hospital.

Immunohistochemistry. Paraffin was removed from the slides by incubation in xylene three times $(10 \mathrm{~min}$ each at room temperature), followed by rehydration in a graded series of ethanol concentrations $(100,95,85$ and $75 \%$ ethanol, $10 \mathrm{~min}$ each at room temperature). Antigen retrieval was performed in $100^{\circ} \mathrm{C}$ water with $0.01 \mathrm{M}$ citrate buffer for $30 \mathrm{~min}$. The sections were incubated at $37^{\circ} \mathrm{C}$ with $0.3 \%$ hydrogen peroxide for $30 \mathrm{~min}$ and blocked at room temperature with $5 \%$ bovine serum albumin (Gibco; Thermo Fisher Scientific, Inc.) for $1 \mathrm{~h}$. Next, the sections were incubated with anti-Rictor antibody (1:800) for $30 \mathrm{~min}$ at $37^{\circ} \mathrm{C}$, followed by incubation with Alexa Fluor ${ }^{\circledR}$ 594-conjugated goat anti-mouse IgG secondary antibody (1:500) at room temperature for $1 \mathrm{~h}$. The slides were then rinsed with PBS-Tween-20 (three times, 5 min each), stained with 3,3'-diaminobenzidine (Thermo Fisher Scientific, Inc.), rinsed with deionized water and counterstained with hematoxylin under a light microscope at x200 magnification. Scoring was conducted based on the staining intensity and the percentage of positively stained cells as follows: $0-5 \%$ scored $0 ; 6-35 \%$ scored $1 ; 36-70 \%$ scored 2 ; and $>70 \%$ scored 3. A final score of $\leq 1$ was assigned to the negative expression group, while a final score of $\geq 2$ was assigned to the positive expression group. Two senior pathologists determined the scores independently.

Cell culture and western blotting. HCT116, SW480, LoVo and HL-7702 (a liver cancer cell line was included to investigate
CRC vs. paracarcinoma, as hepatic metastases are common in patients with CRC) cells were cultured as follows: Each cell line was seeded into 6-well plates at a density of $2.0 \times 10^{5}$ cells/well in triplicate. Cells were grown in $3 \mathrm{ml}$ growth medium $(90 \%$ RPMI- 1640 medium and $10 \% \mathrm{FBS}$ ) at $37^{\circ} \mathrm{C}$, and cell lysis was conducted at $\sim 85 \%$ confluence. HCT116, SW480, LoVo and HL-7702 cells were harvested and lysed following routine methods as previously described (11). Proteins were quantified using the BCA method and $30 \mu \mathrm{g}$ proteins/lane were separated by $6 \%$ SDS-PAGE. Following transfer to a polyvinylidene fluoride membrane, samples were blocked with 5\% FBS at room temperature for $1 \mathrm{~h}$. Subsequently, samples were incubated overnight at $4^{\circ} \mathrm{C}$ with anti-Rictor $(1: 1,000)$ and anti- $\beta$-tubulin $(1: 1,000)$ primary antibodies. Finally, the membrane was incubated with Alexa Fluor ${ }^{\circledR}$ 594-conjugated goat anti-mouse IgG secondary antibody (1:100) and goat anti-mouse $\operatorname{IgG}(1: 100)$ for $30 \mathrm{~min}$ at room temperature. Proteins were visualized using ECL Western Blotting kit (Biyuntian Biotech Co., Ltd.) and In Vivo Imaging System F (Kodak, Rochester, NY, USA).

Statistical analysis. Statistical analyses were conducted using SPSS 20.0 software (IBM SPSS, Armonk, NY, USA). Fisher's exact or $\chi^{2}$ tests were used for the comparison of the Rictor expression rate between groups, while the correlation between survival time and clinicopathological variables was analyzed using the Kaplan-Meier method. For survival analysis, survival curves were compared using the log-rank test. $\mathrm{P}<0.05$ was considered to indicate a statistically significant difference.

\section{Results}

Immunohistochemistry. The immunohistochemical results indicated that Rictor protein mainly existed in the cytoplasm (Fig. 1). The positive expression rate of Rictor in CRC tissues was $58.1 \%$ (36/62), while it was $14.5 \%$ (9/62) in normal tissues adjacent to carcinoma. The expression level of Rictor protein in CRC tissues was significantly higher than that in normal tissues adjacent to colorectal carcinoma $(\mathrm{P}<0.0001)$. The results are shown in Table I.

To investigate the correlation between Rictor expression and clinicopathological characteristics, 62 CRC samples were collected to detect the expression of Rictor protein by immunochemistry. The positive rate of Rictor protein expression and the $\chi^{2}$ values are listed in Table I. Statistical correlation analysis revealed that the expression level of Rictor protein in CRC tissues was associated with Dukes stage $(\mathrm{P}=0.000174)$ 
Table I. Association between Rictor expression and clinicopathological features of CRC patients.

\begin{tabular}{|c|c|c|c|c|c|}
\hline Pathological results & $\mathrm{n}$ & Rictor-negative, $\mathrm{n}(\%)$ & Rictor-positive, n (\%) & $\chi^{2}$ & P-value \\
\hline Tissue & & & & 25.428 & $<0.001$ \\
\hline $\mathrm{CRC}$ & 62 & $26(41.9)$ & $36(58.1)$ & & \\
\hline Normal & 62 & $53(85.5)$ & $9(14.5)$ & & \\
\hline Sex & & & & 0.147 & 0.701 \\
\hline Male & 34 & $15(44.1)$ & $19(55.9)$ & & \\
\hline Female & 28 & $11(39.3)$ & $17(60.7)$ & & \\
\hline Age, years & & & & 0.045 & 0.831 \\
\hline$>65$ & 42 & $18(42.9)$ & $24(57.1)$ & & \\
\hline$<65$ & 20 & $8(40.0)$ & $12(60.0)$ & & \\
\hline Differentiation degree & & & & 0.421 & 0.516 \\
\hline High & 21 & $10(47.6)$ & $11(52.4)$ & & \\
\hline Low & 41 & $16(39.0)$ & $25(61.0)$ & & \\
\hline Tumor size, cm & & & & 0.047 & 0.829 \\
\hline$>3$ & 32 & $13(40.6)$ & $19(59.4)$ & & \\
\hline$<3$ & 30 & $13(43.3)$ & $17(56.7)$ & & \\
\hline Depth of invasion & & & & 0.633 & 0.426 \\
\hline Muscular layer & 25 & $12(48.0)$ & $13(52.0)$ & & \\
\hline Serosal layer & 37 & $14(37.8)$ & $23(62.2)$ & & \\
\hline Lymphatic metastasis & & & & 14.600 & $<0.001$ \\
\hline With & 32 & $6(18.8)$ & $26(81.2)$ & & \\
\hline Without & 30 & $20(66.7)$ & $10(33.3)$ & & \\
\hline Dukes stage & & & & 14.090 & $<0.001$ \\
\hline $\mathrm{A}+\mathrm{B}$ & 28 & $19(67.9)$ & $9(32.1)$ & & \\
\hline $\mathrm{C}+\mathrm{D}$ & 34 & $7(20.6)$ & $27(79.4)$ & & \\
\hline
\end{tabular}

Rictor, rapamycin-insensitive companion of mammalian target of rapamycin; CRC, colorectal cancer.

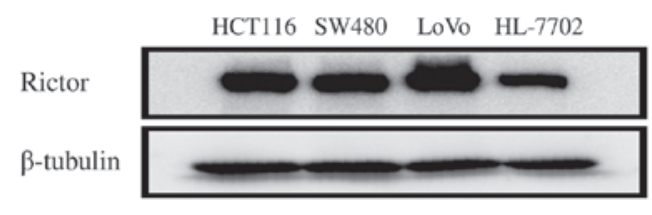

Figure 2. Western blotting results. The expression of Rictor in colorectal cancer cell lines (HCT116, SW480 and LoVo) was higher than that in a normal liver cell line (HL-7702). Rictor, rapamycin-insensitive companion of mammalian target of rapamycin.

and lymph node metastasis $(\mathrm{P}=0.000133)$, but not with patients' age, sex, tumor size, differentiation degree, depth of invasion or histological type $(\mathrm{P}>0.05)$. The results are presented in Table I.

Western blotting. In order to identify the expression and significance of Rictor protein in CRC at the cellular level, Rictor protein expression was firstly detected in HCT116, SW480, LoVo and HL-7702 cells using western blotting. Rictor protein expression in these CRC cell lines was higher than that in the normal liver cell line HL-7702 (Fig. 2). Thus, these in vitro results also suggested that Rictor overexpression existed in CRC, which implies that Rictor may be involved in tumorigenesis and cancer progression in CRC.

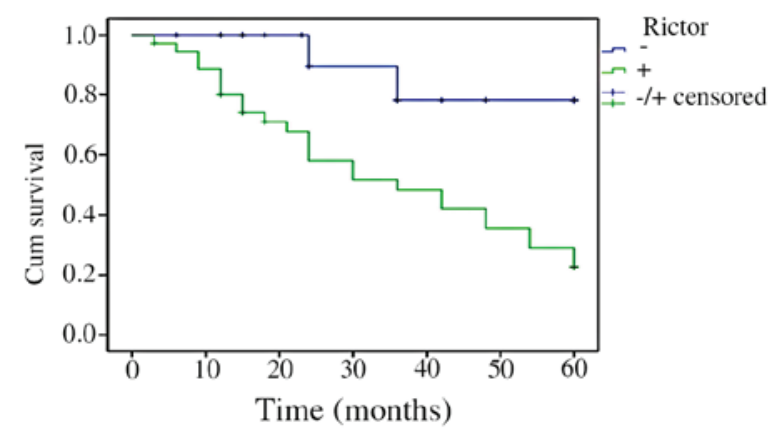

Figure 3. Survival analysis results. Kaplan-Meier test revealed an association between Rictor protein expression and patients' survival time. Rictor, rapamycin-insensitive companion of mammalian target of rapamycin; Cum, cumulative.

Rictor protein expression and survival analysis. The Kaplan-Meier survival analysis results indicated that the survival time of patients with CRC was correlated with Rictor protein expression levels. Patients whose Rictor protein expression was positive had a shorter survival time than those with negative expression. Log-rank test revealed that the difference was statistically significant $(\mathrm{P}=0.000415)$. The results are shown in Fig. 3. 


\section{Discussion}

The initiation and progression of CRC is a multi-step process involving gradual changes. Numerous genes are closely associated with the progression of CRC. Therefore, identifying therapeutic targets for CRC and improving the 5-year survival rate of patients with $\mathrm{CRC}$ has important theoretical and practical significance. Rictor, as a member of mTORC2, is important in the process of tumor proliferation, migration, invasion, epithelial-mesenchymal transition (EMT) and poor prognosis (12-16). For example, Akt activated by Rictor phosphorylation induced the expression of c-Myc and cyclin E, and this process could regulate the proliferation and cell cycle of colon cancer cells. Blocking Rictor phosphorylation, which was able to lessen the inhibition of the mTORC2-Akt signaling pathway, was applied to stop tumor progression and cancer cell proliferation (17). Bashir et al reported that the Rictor gene was involved in cancer cell proliferation in malignant glioma and had a high recurrence rate in hepatic carcinoma (18). A previous study indicated that integrin-linked kinase (ILK), a focal adhesion adaptor, is an STK that regulates cell proliferation, survival and EMT. The ILK-Rictor complex acts as a potential molecular target for preventing/reversing fibrosis caused by EMT, cancer progression and metastasis (13). Tsuruta et al observed that Rictor, as a target gene of miR-152, participated in regulating the proliferation of cancer cells in endometrial carcinoma (10). Therefore, it was hypothesized that Rictor protein may be involved in the proliferation, migration and invasion of CRC.

Based on this theory, the expression level of Rictor protein was analyzed in $62 \mathrm{CRC}$ tissues. The results indicated that Rictor expression in CRC tissues was markedly higher than that in adjacent normal tissues. Western blotting results also confirmed this finding at the cellular level, suggesting that overexpression of Rictor protein existed in CRC, which may participate in the occurrence and development of CRC. However, the specific biological function of Rictor is still unknown. It has been reported recently that the upregulation of Rictor is associated with an increased rate of relapse in hepatocellular carcinoma. Through analysis of the correlation between Rictor protein and clinicopathological parameters, the present study further confirmed that Rictor protein had a significant correlation with Dukes stage and lymph node metastasis, which further verified our original experimental hypothesis. Thus, it could be speculated that Rictor has the potential to be one of the new targets of oncotherapy and may be applied to clinical diagnosis, similarly to other tumor markers.

In the present study, Kaplan-Meier survival analysis was used to evaluate the association between the survival time of patients with CRC and Rictor protein expression level. The results revealed that positive expression of Rictor protein in patients with CRC led to a shorter overall survival compared with that in patients exhibiting negative expression, indicating that Rictor protein can be used as a prognostic indicator of CRC. A previous study also revealed that Rictor overexpression may result in ovarian cancer patients becoming resistant to Taxol (17), which suggests that targeted control of Rictor in cancer therapy may be used to improve drug sensitivity. Several adenosine triphosphate-competitive and selective
mTOR inhibitors, which target simultaneously mTORC1 and mTORC2, have been developed (19-21). A previous study demonstrated that the anti-proliferative efficacy of these inhibitors is superior to that of rapamycin (20). However, if these inhibitors had larger toxicity in vivo than rapamycin, this could be a major concern. Another study also suggested that targeting only mTORC2 was sufficient to prevent colon cancer progression (22).

Future studies may provide a rational answer about whether drugs that specifically target Rictor could be used to treat CRC or other malignant tumors. CRC has been treated with rapamycin for a long period of time; however, the clinical results are discouraging (23). One proposed mechanism of resistance to rapamycin is the inability of this drug to inhibit mTORC2 $(24,25)$. It has been reported that Rictor contributed to cisplatin resistance in human ovarian cancer cells (26). This supports the hypothesis that mTORC2 compensates for the loss of mTORC1 activity upon rapamycin treatment, thereby leading to rapamycin resistance. Knockdown of Rictor was reported to induce G1 arrest in the breast cancer cell line MCF7 and in the prostate cancer cell line PC3 (27). In addition, inhibition of the Akt-mTOR signaling pathway was shown to induce G2-M arrest and autophagy in the breast cancer cell line MDA-MB-231 (28). Considering the present results, it can be proposed that Rictor may be key to improve the therapeutic regimen for treating CRC patients.

In summary, the present study demonstrated that Rictor expression may be associated with CRC initiation and progression, and also confirmed that Rictor expression is associated with Dukes stage and lymph node metastasis. Additionally, it was further verified that Rictor had a correlation with the prognosis of patients with CRC. Thus, Rictor could play a crucial role in directing treatment and prognosis evaluation in CRC patients.

\section{Acknowledgements}

The authors thank Professor Zhao Liang (Department of Pathology, Southern Medical University, Guangzhou, China) for providing useful guiding ideas and discussions.

\section{References}

1. Siegel RL, Miller KD and Jemal A: Cancer statistics, 2015. CA Cancer J Clin 65: 5-29, 2015.

2. Chiarini F, Evangelisti C, McCubrey JA and Martelli AM: Current treatment strategies for inhibiting mTOR in cancer. Trends Pharmacol Sci 36: 124-135, 2015.

3. Iwaya T, Yokobori T, Nishida N, Kogo R, Sudo T, Tanaka F, Shibata K, Sawada G, Takahashi Y, Ishibashi M, et al: Downregulation of miR-144 is associated with colorectal cancer progression via activation of mTOR signaling pathway. Carcinogenesis 33: 2391-2397, 2012.

4. Lee DF, Kuo HP, Chen CT, Hsu JM, Chou CK, Wei Y, Sun HL, Li LY, Ping B, Huang WC, et al: IKK beta suppression of TSC1 links inflammation and tumor angiogenesis via the mTOR pathway. Cell 130: 440-455, 2007.

5. Sarbassov DD, Ali SM, Kim DH, Guertin DA, Latek RR, Erdjument-Bromage H, Tempst P and Sabatini DM: Rictor, a novel binding partner of mTOR, defines a rapamycin-insensitive and raptor-independent pathway that regulates the cytoskeleton. Curr Biol 14: 1296-1302, 2004.

6. Jacinto E, Loewith R, Schmidt A, Lin S, Rüegg MA, Hall A and Hall MN: Mammalian TOR complex 2 controls the actin cytoskeleton and is rapamycin insensitive. Nat Cell Biol 6: 1122-1128, 2004. 
7. Sarbassov DD, Guertin DA, Ali SM and Sabatini DM: Phosphorylation and regulation of Akt/PKB by the rictor-mTOR complex. Science 307: 1098-1101, 2005.

8. Jacinto E, Facchinetti V, Liu D, Soto N, Wei S, Jung SY, Huang Q, Qin J and Su B: SIN1/MIP1 maintains rictor-mTOR complex integrity and regulates Akt phosphorylation and substrate specificity. Cell 127: 125-137, 2006.

9. Pearce LR, Huang X, Boudeau J, Pawłowski R, Wullschleger S, Deak M, Ibrahim AF, Gourlay R, Magnuson MA and Alessi DR Identification of Protor as a novel Rictor-binding component of mTOR complex-2. Biochem J 405: 513-522, 2007.

10. Tsuruta T, Kozaki K, Uesugi A, Furuta M, Hirasawa A, Imoto I, Susumu N, Aoki D and Inazawa J: miR-152 is a tumor suppressor microRNA that is silenced by DNA hypermethylation in endometrial cancer. Cancer Res 71: 6450-6462, 2011.

11. McDonald PC, Oloumi A, Mills J, Dobreva I, Maidan M, Gray V, Wederell ED, Bally MB, Foster LJ and Dedhar S: Rictor and integrin-linked kinase interact and regulate Akt phosphorylation and cancer cell survival. Cancer Res 68: 1618-1624, 2008

12. Agarwal NK, Chen CH, Cho H, Boulbès DR, Spooner E and Sarbassov DD: Rictor regulates cell migration by suppressing RhoGDI2. Oncogene 32: 2521-2526, 2013.

13. Serrano I, McDonald PC, Lock FE and Dedhar S: Role of the integrin-linked kinase (ILK)/Rictor complex in TGF $\beta$-1-induced epithelial-mesenchymal transition (EMT). Oncogene 32: 50-60, 2013.

14. Verreault M, Weppler SA, Stegeman A, Warburton C, Strutt D, Masin D and Bally MB: Combined RNAi-mediated suppression of Rictor and EGFR resulted in complete tumor regression in an orthotopic glioblastoma tumor model. PLoS One 8: e59597, 2013.

15. Oneyama C, Kito Y, Asai R, Ikeda J, Yoshida T, Okuzaki D, Kokuda R, Kakumoto K, Takayama K, Inoue S, et al: miR-424/503-mediated Rictor upregulation promotes tumor progression. PLoS One 8: e80300, 2013.

16. Zhang F, Zhang X, Li M, Chen P, Zhang B, Guo H, Cao W, Wei X, Cao X, Hao X and Zhang N: mTOR complex component Rictor interacts with PKCzeta and regulates cancer cell metastasis. Cancer Res 70: 9360-9370, 2010.

17. Chen CH, Shaikenov T, Peterson TR, Aimbetov R, Bissenbaev AK, Lee SW, Wu J, Lin HK and Sarbassov dos D: ER stress inhibits mTORC 2 and Akt signaling through GSK-3 $\beta$-mediated phosphorylation of rictor. Sci Signal 4: ra10, 2011.
18. Bashir T, Cloninger C, Artinian N, Anderson L, Bernath A, Holmes B, Benavides-Serrato A, Sabha N, Nishimura RN, Guha A and Gera J: Conditional astroglial Rictor overexpression induces malignant glioma in mice. PLoS One 7: e47741, 2012.

19. Feldman ME, Apsel B, Uotila A, Loewith R, Knight ZA, Ruggero D and Shokat KM: Active-site inhibitors of mTOR target rapamycin-resistant outputs of mTORC1 and mTORC2. PLoS Biol 7: e38, 2009.

20. Yu K, Toral-Barza L, Shi C, Zhang WG, Lucas J, Shor B, Kim J, Verheijen J, Curran K, Malwitz DJ, et al: Biochemical, cellular, and in vivo activity of novel ATP-competitive and selective inhibitors of the mammalian target of rapamycin. Cancer Res 69: 6232-6240, 2009.

21. Thoreen CC, Kang SA, Chang JW, Liu Q, Zhang J, Gao Y, Reichling LJ, Sim T, Sabatini DM and Gray NS: An ATP-competitive mammalian target of rapamycin inhibitor reveals rapamycin-resistant functions of mTORC1. J Biol Chem 284: 8023-8032, 2009.

22. Roulin D, Cerantola Y, Dormond-Meuwly A, Demartines N and Dormond O: Targeting mTORC2 inhibits colon cancer cell proliferation in vitro and tumor formation in vivo. Mol Cancer 9: 57, 2010.

23. Faivre S, Kroemer G and Raymond E: Current development of mTOR inhibitors as anticancer agents. Nat Rev Drug Discov 5: 671-688, 2006.

24. Guertin DA and Sabatini DM: Defining the role of mTOR in cancer. Cancer Cell 12: 9-22, 2007.

25. Gulhati P, Cai Q, Li J, Liu J, Rychahou PG, Qiu S, Lee EY, Silva SR, Bowen KA, Gao T and Evers BM: Targeted inhibition of mammalian target of rapamycin signaling inhibits tumorigenesis of colorectal cancer. Clin Cancer Res 15: 7207-7216, 2009.

26. Im-aram A, Farrand L, Bae SM, Song G, Song YS, Han JY and Tsang BK: The mTORC2 component rictor contributes to cisplatin resistance in human ovarian cancer cells. PLoS One 8: e75455, 2013

27. Hietakangas V and Cohen SM: TOR complex 2 is needed for cell cycle progression and anchorage-independent growth of MCF7 and PC3 tumor cells. BMC Cancer 8: 282, 2008.

28. Kuo PL, Hsu YL and Cho CY: Plumbagin induces G2-M arrest and autophagy by inhibiting the AKT/mammalian target of rapamycin pathway in breast cancer cells. Mol Cancer Ther 5: 3209-3221, 2006. 